\title{
Low Voltage Distribution Network Line Loss Calculation Based on The Theory of Three- phase Unbalanced Load
}

\author{
HU Jinhong ${ }^{1, a,{ }^{*},}$, FU Xiaofei ${ }^{1}$,LIAO Tianming ${ }^{1}$,CHEN Xin ${ }^{1}$,JI Kunhua ${ }^{1}$,SHENG Hui ${ }^{1}$, and \\ ZHAO Wenbin ${ }^{2}$ \\ ${ }^{1}$ Shanghai Electric Power Company,Shanghai, China \\ ${ }^{2}$ Shanghai University of Electric Power, Shanghai, China \\ a zengyan2511@163.com \\ *corresponding author
}

Keywords: low-voltage distribution, equivalent resistance calculation method, three-phase unbalanced load

\begin{abstract}
Based on the operation characteristics of the rural public low-voltage distribution transformer areas, this paper puts forward the theoretical calculation math model and the equivalent resistance calculation method of line loss, in which the unbalanced three-phase load loss coefficient is considered as the main factor. Compared to the traditional estimation method, it greatly improves the accuracy of theoretical calculation of the line loss in these areas. In this paper, combined with the structure and power consumption characteristics of the low pressure platform, a regional representative area is selected for the research. The regional and seasonal loads of the selected stations are analysed. Finally, a stage area of theoretical line loss calculation that takes into account the three-phase unbalanced load.
\end{abstract}

\section{Introduction}

The classical algorithm of distribution network theoretical line loss is equivalent resistance method. However, the equivalent resistance method is suitable for the calculation of the line loss of the $10 \mathrm{kV}$ public distribution network with relatively single and three-phase load balance ${ }^{[1]}$. It is not suitable for the low voltage power grid (hereinafter referred to as the low pressure area) of the rural public transformer platform ${ }^{[2]}$. The main reason is the low pressure platform structure and load characteristics and $10 \mathrm{kV}$ common lines are very different.

From the power supply mode, $10 \mathrm{kV}$ public line only three-phase-three-wire system, the one power supply. But the low-pressure platform there are three-phase-four-wire trunk lines and branch lines, three-phase-three-wire power load branch line and the line ${ }^{[3]}$, low-voltage single-phase-twoline residents living lighting branch line and the line of three kinds of power supply. From the load characteristics, the three-phase load $10 \mathrm{kV}$ public distribution network is more balanced, and the low load station area, especially the rural public low-voltage three-phase load is not balanced ${ }^{[4]}$. The 
unbalance of the three-phase load is much higher than that of the $10 \mathrm{kV}$ line, and the magnitude and phase of the zero current of the main line and the branch line of the three-phase-four wire system change with the variation of the three-phase load imbalance ${ }^{[4]}$. Three phase load unbalance is one of the main factors that affect the line loss in low voltage area, and also is one of the main factors that affect the calculation accuracy of line loss in low voltage area. It is helpful to improve the accuracy of the theoretical line loss calculation of the low pressure station and solve the problem of the accuracy of the traditional rough estimation algorithm by introducing the unbalanced loss coefficient into the low voltage station equivalent resistance calculation.

\section{Analysis of unbalanced three-phase load current}

Three phase unbalance is defined as follows ${ }^{[5]}$ :

$$
\delta_{h}=\frac{I_{h \max }-I_{p j h}}{I_{p j h}} \times 100 \%
$$

In the formula, $I_{h \max }$ represents the maximum value of the three-phase current for one hour, $I_{p j h}=\left(I_{a h}+I_{b h}+I_{c h}\right) / 3$, and $I_{a h} 、 I_{b h} 、 I_{c h}$ represent the three-phase current per hour respectively.

Figure 1 to 2, respectively, given the three-phase current curve of the selected representative day from NanXin2, and XiaNan post office. Figure 3 to 4, given the three-phase unbalance curve of the selected representative day from NanXin2, XiaNan post office, Dongjin Gardening and SunQiao home. Maximum three-phase imbalance up to $97 \%$.

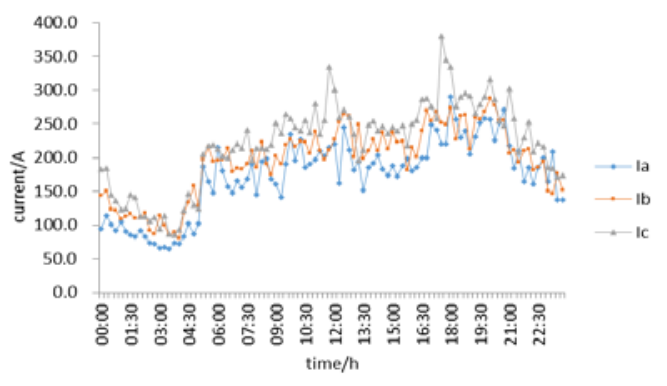

Figure.1 three phase current curve of working day (NanXin2, 6th June)

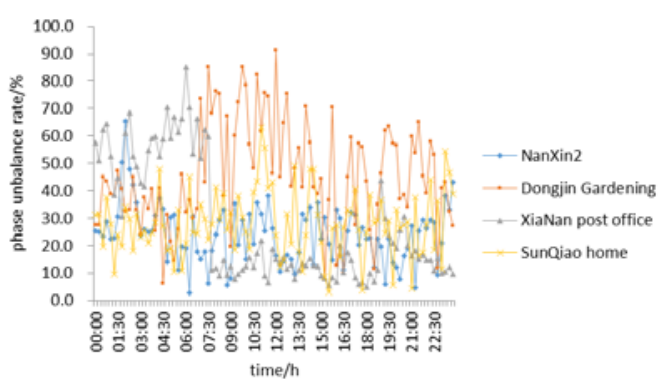

Figure. 3 three-phase unbalance curve of working day (13th July)

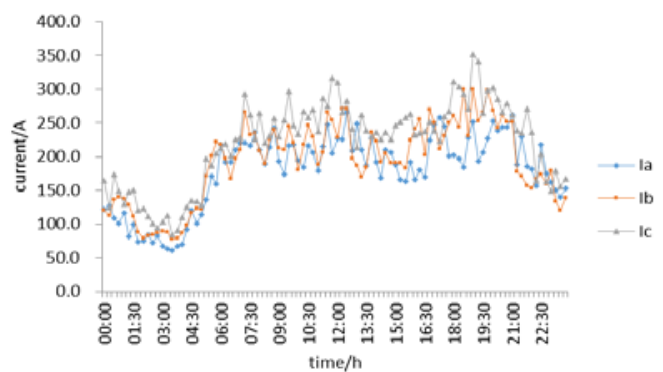

Figure.2 three phase current curve of holiday (XiaNan post office, 1th May)

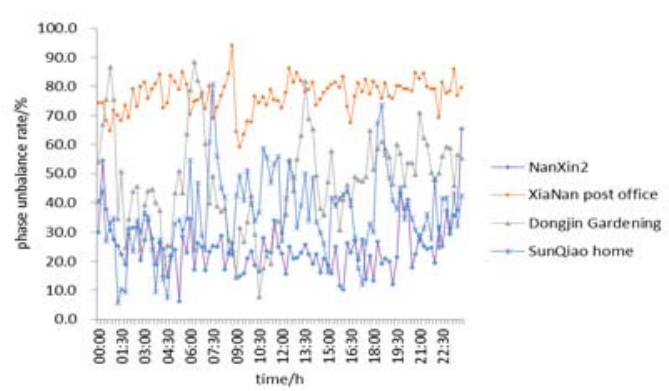

Figure. 4 three-phase unbalance curve of holiday (2th May)

\section{Loss algorithm of line loss based on three - phase load unbalance}

When the three-phase load is unbalanced, there is a current on the zero line to produce zero line power loss, so that the three-phase four-wire system produces the total power loss greater than the three-phase four-wire balanced power supply line. 
Assume that $I_{A}>I_{B}>I_{C}, I_{o} \neq 0, \beta_{1}=I_{B} / I_{A}, \beta_{2}=I_{C} / I_{A}, \beta_{1}, \beta_{2}$ size reflects the imbalance of the three-phase load current. Suppose $\alpha$ is a loss coefficient of unbalance. Indicating that the threephase four-wire system in the three-phase load current imbalance generated when the theoretical line loss value relative to the three-phase equilibrium when the theoretical loss of multiple. Theoretical projections can be obtained ${ }^{[5]}$ :

$$
\alpha=\frac{1+\beta_{1}^{2}+\beta_{2}^{2}+\left(1-\frac{\beta_{1}+\beta_{2}}{2}\right)}{\frac{\left(1+\beta_{1}+\beta_{2}\right)^{2}}{2}}
$$

(1)When $I_{A}=I_{B}=I_{C}$, that is, when the three-phase load is fully balanced, $\beta_{1}=\beta_{2}=1, \alpha=1$, indicating that the three-phase four-wire balanced power supply and three-phase three-wire power supply equal to the theoretical line loss.

(2)When $I_{A} \neq 0, I_{B}=I_{C}=0$, that is, three-phase four-wire power supply into a single-phase power supply this pole balance state, $\beta_{1}=\beta_{2}=0, \alpha=6$, indicating that the load size and line technical parameters in the same conditions, three-phase four-wire balanced power supply line loss is single-phase two-wire power supply theory line loss of 6 times.

$\alpha$ in the formula (2-1) is introduced into the three-phase line loss calculation formula, the threephase four-wire line power loss can be expressed as:

$$
\Delta P=3 \alpha(K I)^{2} R \times 10^{-3}=3 \alpha I^{2} R \times 10^{-3}
$$

\section{Calculation of theoretical line loss by equivalent resistance method}

\subsection{The basic principles}

The basic idea of calculating the line loss by the equivalent resistance method is: at the beginning of the low-voltage distribution line is assumed to have an equivalent line resistance Req, the average current at the head of the line flows through the losses generated by Req. The current through each branch line loss caused by the resistance equal to the sum of the respective branch.

The equivalent resistance method is relatively simple to use, its accuracy can meet the practical requirements, and it is a more practical low-voltage distribution network theoretical line loss calculation method. This method assumes that the load power factor, load coefficient and voltage is the same, but these values are not the same in the actual system.

\subsection{The basic steps}

(a) Calculation of line loss

(1) Single-phase-two-wire system

$$
\Delta A=2 I_{a v}^{2} K^{2} R_{\mathrm{eq}} T \times 10^{-3}(\mathrm{kWh})
$$

(2) Three-phase-three-wire system

$$
\Delta A=3 I_{a v}^{2} K^{2} R_{\mathrm{eq}} T \times 10^{-3}(\mathrm{kWh})
$$

(3) Three-phase-four-wire system

$$
\Delta A=3 \alpha I_{a v}^{2} K^{2} R_{\text {eq }} T \times 10^{-3}(\mathrm{kWh})
$$


In the formula, $I_{a v}--$ the average load current at the head of the low voltage network, A;

$T$-- low voltage network power supply time, h;

$K$-- wave coefficient, the same value with the high voltage network;

Req-- low voltage equivalent resistance, $\Omega$;

$\alpha$-- unbalanced loss coefficient.

(b) Calculation of average current $I_{a v}$

If the distribution transformer capacity Se is greater than $100 \mathrm{kVA}$, the secondary side of the distribution transformer is equipped with active and reactive energy meter. The average current $I_{a v}$ of the three-phase line can be calculated by the following equation:

$$
I_{a v}=\frac{1}{U_{a v} T} \sqrt{\frac{1}{3}\left(A_{Y G}^{2}+A_{W G}^{2}\right)}
$$

If the secondary outlet of the distribution transformer is only equipped with a power meter and a power factor meter, then:

$$
I_{a v}=\frac{A_{Y G}}{\sqrt{3} U_{a v} \cos \varphi T}
$$

In the formula, $A_{Y G}-$ the active power at the head of the low voltage network, $\mathrm{kWh}$;

$A_{w G}$-- the reactive power at the head of the low voltage network, kVarh;

$U_{a v}{ }^{--}$average operating voltage, advisable $0.38 \mathrm{kV}$;

$T$-- operation hours, h;

(c) Calculation of equivalent resistance Req

Before calculation, the low-voltage lines are divided into several calculation lines from the end to the head end, and from the branch line to the trunk line. The principle of segmentation is: where the power load transmission, the transmission line number used, and the length of the line are the same line, it is considered the same line. Otherwise the other as a calculation line segment. Then, the formula of Req is:

$$
R_{e q}=\frac{\sum_{i=1}^{n} N_{i} A_{i}^{2} R_{i}}{3.5\left(\sum_{i=1}^{n} A_{i}\right)^{2}}
$$

In the formula, $A_{i}--$ the electric of meter reading fron the $380 / 220 \mathrm{~V}$ user power meter, $\mathrm{kWh}$;

$$
\begin{aligned}
& A_{i \sum} \text {--the sum of meter reading of low voltage user which power supply by a } \\
& \text { segment, } \mathrm{kWh} \text {; } \\
& R_{i} \text {-- the wire resistance of line } i \text {; } \\
& N_{i} \text {-- the structural constants of line } i \text {, single-phase two-wire system } N=2 \text {, three- } \\
& \quad \text { phase three-wire } N=2 \text {, three-phase four-wire } N=3 \alpha \text {. }
\end{aligned}
$$




\section{Case study}

\subsection{The calculation steps}

Equivalent resistance method for calculating the theoretical practice of low pressure zone, the calculation steps are as follows:

(1) Draw out the wiring diagram of the network, and divide the line calculation line segment from the trunk line and branch line, and then calculate the load power by paragraph.

(2) Calculate the line segment resistance and line equivalent resistance.

(3) Calculate the average load current at the head of the line.

(4) Real-time measurement of the three-phase current of each calculated line segment, and calculate the unbalance loss coefficient of each line segment.

(5) Calculate the characteristic curve of the load curve of the line.

(6) Check the actual power supply time and the meter reading power of distribution transformer to low voltage line from the use of electricity or the record of operation.

(7) Substituting parameter value that obtained by the determination, retrieval and calculation into formula, calculating the theoretical line loss of low voltage distribution network.

\subsection{The calculation examples}

A $380 / 220 \mathrm{~V}$ low-voltage distribution lines, month running time $\mathrm{T}=537 \mathrm{~h}$, active power supply $A_{Y G}=3542 k W h, K=1.16, \cos \varphi=0.85$. The line structure is shown in figure 5 , the electricity consumption of each branch line (the actual values, $\mathrm{kWh}$ ) and resistances (the actual values, $\Omega$ ) are marked in the figure.

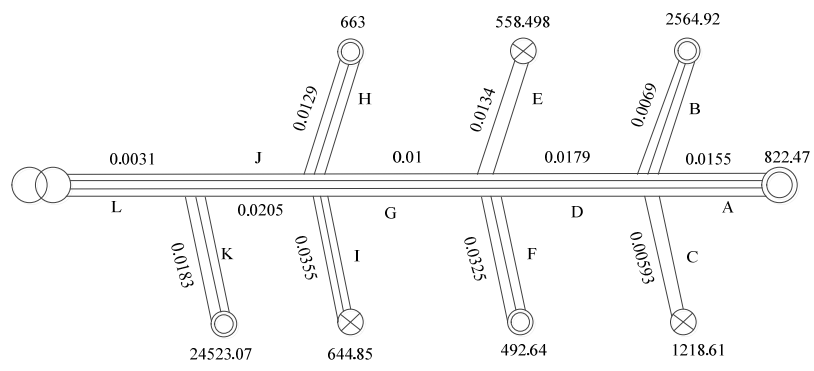

Figure.5 The diagram of line structure

(1)The average current is

$$
\begin{aligned}
I_{a v} & =\frac{A_{Y G}}{\sqrt{3} U_{e} \cos \varphi T} \\
& =\frac{35424}{\sqrt{3} \times 380 \times 0.85 \times 537}=117.91
\end{aligned}
$$

(2)The denominator value of the equivalent resistance is

$$
\begin{aligned}
3.5\left(\sum_{i=1}^{m} A_{i}\right)^{2}= & 3.5 \times(24523.07+663+644.85+558.49 \\
& +492.64+2564.92+1218.61+822.47)^{2} \\
= & 3.5 \times 31488.05^{2}
\end{aligned}
$$


(3) The molecular calculation of the equivalent resistance is

Line resistance of $\mathrm{A}, R_{\mathrm{A}}=3 * 1.65 * 822.4^{\wedge} 2 *(0.0155)$

Line resistance of $\mathrm{B}, R_{\mathrm{B}}=3 * 2564.92^{\wedge} 2 * 0.0069$

Line resistance of C, $R_{\mathrm{C}}=2 * 1218.61^{\wedge} 2 * 0.00593$

Line resistance of $\mathrm{D}, R_{\mathrm{D}}=3 * 1.53 *(2564.92+1218.61+822.47) \wedge 2 * 0.0179$

Line resistance of $\mathrm{E}, R_{\mathrm{E}}=2 * 558.49^{\wedge} 2 * 0.0134$

Line resistance of F, $R_{\mathrm{F}}=3 * 492.64 \wedge 2 * 0.0325$

Line resistance of $\mathrm{G}, R_{\mathrm{G}}=3 * 1.65 *(558.49+492.64+2564.92+1218.61+822.47)^{\wedge} 2 * 0.0100$

Line resistance of H. $R_{\mathrm{H}}=3^{*} 663^{\wedge} 2 * 0.0129$

Line resistance of I. $R_{\mathrm{I}}=3 * 644.85^{\wedge} 2 * 0.0355$

Line resistance of $\mathrm{J}, R_{\mathrm{J}}=3 * 1.67 *(644.85+558.49+492.64$

$+2564.92+1218.61+822.47)^{\wedge} 2 * 0.0205$

Line resistance of K. $R_{\mathrm{K}}=3 * 24523.07^{\wedge} 2 * 0.0183$

Line resistance of K. $R_{\mathrm{L}}=3 * 1.6^{*} 31488.05^{\wedge} 2^{*} 0.0031$

The molecular value of the equivalent resistance is

$$
\begin{aligned}
\sum_{i}^{n} N_{i} A_{i}^{2} R_{i}= & R_{A}+R_{B}+R_{C}+R_{D}+R_{E}+R_{E} \\
& +R_{G}+R_{H}+R_{I}+R_{J}+R_{K}+R_{L}
\end{aligned}
$$

The equivalent resistance is:

$$
R_{e q}=\frac{\sum_{i=1}^{n} N_{i} A_{i \sum}^{2} R_{i}}{N\left(\sum_{i=1}^{m} A_{i}\right)^{2}}=0.01599(\Omega)
$$

(4) The loss of electricity in low voltage line

$$
\Delta A=3.5 I_{a v}^{2} K^{2} R_{e q} T \times 10^{-3}=761.28(\mathrm{kWh})
$$

(5) Theoretical line loss rate

$$
\Delta A(\%)=\frac{\Delta A}{A_{Y G}} \times 100 \%=2.15 \%
$$

(6) Statistical line loss rate

$$
\Delta A_{S}(\%)=\frac{A_{Y G}-\sum_{i=1}^{n} A_{i}}{A_{Y G}} \times 100 \%=11.09 \%
$$

As can be seen from the above equation, the low-voltage theoretical line loss in this area is less to statistical line loss. This shows that the management loss of the area to occupy a very large proportion compared with line loss. Thus, bringing inspiration to the power sector, and it is necessary for the power sector to pay attention to strengthen management. In order to reduce the measurement equipment by the error, mismanagement and mistakes caused by wire damage, And to prevent the line loss due to electric larceny, reading wrong and lack in meter reading. At the same time, the three-phase current unbalance in the low-pressure area of the region is serious. Taking effective measures to weaken the impact of three-phase imbalance is necessary. 


\section{Conclusion}

In this paper, we studied the three-phase load current imbalance of low-voltage distribution network, and considered the three-phase load imbalance conditions, used equivalent resistance method to solve the problem of theoretical line loss of low pressure zone. It provides a reference for the theoretical line loss calculation of low pressure station.

\section{References}

[1] Zhang Ming, Xie Shanshan, Luo Yunfeng.(2015) Optimization model of the three-phase load imbalance in low voltage distribution network. Journal of Wuhan University of Science and Technology, 38(1), 59-62.

[2] Zhang Peng, Luo Jian, Ji Chang, Wang Junjie. (2015) Three-phase unbalanced load parameters identification of low voltage power utilization network. Power System Protection and Control, 43(24), 90-95.

[3] Zhao Dongyuan, Gao Yuechun. (2016) Study on Unbalanced Mechanism of Low Voltage Load and Its Control Measures in Distribution Network. Power Capacitor \& Reactive Power Compensation, 37(6), 59-63.

[4] Miao Wei, Ren Siyuan, Pang Yingjun, Zhang Guoqing, Li Yanjun. (2017) Study on Multi-objective Optimal Regulation Strategy Based on Three-phase Unbalance in Distribution Network. Smart Grid, 5(1), 69-74.

[5] Fang Xianghui. (2011) Calculation of Line Loss for Public Low-voltage Transformer Areas Based onUnbalanced Three-phase Load. Rural power grid, 9, 57-60. 